\title{
Low Reynolds Number Surge Response of a Flat Plate Wing at 90 Degrees Incidence
}

\author{
Simon J. Corkery*, P. R. R. J. Stevens ${ }^{\dagger}$ \\ Holger Babinsky \\ Department of Engineering, University of Cambridge, United Kingdom
}

\begin{abstract}
This article presents an experimental investigation into the unsteady force response of a flat plate wing when accelerated from rest at $90^{\circ}$ incidence to the free stream direction. The work was performed to test a low order model developed by the NATO AVT-202 task group for pitching and surging wings at low Reynolds numbers. The model expresses the force response on a wing as the sum of circulatory components due to vortex dynamics, and non-circulatory components due to added mass. Force measurements, flow visualisation, and PIV measurements were taken for a fast and slow acceleration rate to relatively isolate the effect of added mass. Measurements suggest that the modified Wagner function can give a surprisingly reasonable prediction of vortex growth during the acceleration phase of a surging wing, however, incorrectly predicts vortex growth at steady state. It is proposed that added mass is a mechanism that generates vorticity, thus PIV measurements of circulation of the vortex pair can also contain the impulse due to added mass. When PIV measurements are compensated for added mass, good agreement between the drag predicted by the low order model and force balance measurement is shown.
\end{abstract}

\section{Nomenclature}

c Chord length

$\alpha \quad$ Angle of incidence

$U \quad$ Instantaneous stream wise velocity $(\mathrm{m} / \mathrm{s})$

$U_{\infty} \quad$ Steady state stream wise velocity $(\mathrm{m} / \mathrm{s})$

$F_{\text {circ }}^{\prime} \quad$ Circulatory force per unit length (N)

$F_{n o n-c i r c}^{\prime} \quad$ Non circulatory force per unit length (N)

$C_{n o n-c i r c} \quad$ Non circulatory force coefficient

$C_{\text {circ }} \quad$ Circulatory force coefficient

$C_{D} \quad$ Combined drag force coefficient

$\rho \quad$ Density $\left(\mathrm{kg} / \mathrm{m}^{3}\right)$

$\Gamma \quad$ Circulation $\left(\mathrm{m}^{2} / \mathrm{s}\right)$

$d \quad$ Absolute distance between vortex centres (m)

$J \quad$ Impulse (Ns)

$s \quad$ Wing position $(\mathrm{m})$

\section{Introduction}

In recent years there has been interest in developing Micro Air Vehicles (MAV), particularly for the application of military surveillance. ${ }^{1}$ Such vehicles operate at low Reynolds numbers, with unsteady flow conditions that are often highly separated and typically feature strong leading and trailing edge vortices (LEV and TEV respectively). Recently the NATO AVT-202 'Advances in Fundamental Unsteady Low

*Ph.D., sjc276@cam.ac.uk.

${ }^{\dagger}$ Research Associate, Cambridge University Engineering Department, robbie.stevens@cantab.net, AIAA Member.

‡Professor, hb@eng.cam.ac.uk, AIAA Associate Fellow. 
Reynolds Number Flows' task group ${ }^{2-4}$ proposed a low order model developed to understand the physics of low Reynolds number lift generation on wings in unsteady motion. The model deconstructs the force response of the wing into circulatory (bound circulation or growth and advection of a LEV and TEV pair) and non-circulatory (virtual or added mass) contributions. This will be described in detail in section A. Two canonical test cases used for validation of the model were the 'surge' and 'pitch' cases. The surge case was defined as the acceleration of a wing from rest at an angle of incidence of $45^{\circ}$, followed by steady state translation. The pitch case is defined as a rapid change in angle of incidence from 0 to $45^{\circ}$ while in steady translation. Under the assumption of zero bound circulation, for the surge case Babinsky et al. ${ }^{3}$ showed that Wagner's function, when scaled by the instantaneous velocity to correct for a non impulsive start, gave a reasonable fit to experimental measurements for the circulation of the LEV and TEV pair. It was assumed that steady state lift was equivalent to $C_{l}=2 \pi \alpha$, which was related to circulation with the Kutta-Joukowski theorem. The Wagner function describes the growth of bound circulation for an impulsively started aerofoil at low incidence. Vorticity shed into a planar wake reduces the effective angle of incidence of the wing, retarding the growth of bound circulation. It is unknown why the Wagner function reasonably describes the growth of the LEV and TEV for surging and pitching wings at $45^{\circ}$ incidence, as assumptions of the model are clearly void. To explore this further we are considering the case of a surging wing at $90^{\circ}$ incidence (accelerating normal to the surface). Prior experiments on accelerating plate sections by Pierce, ${ }^{5}$ Pullin and Perry, ${ }^{6}$ as well as Lian and Huang ${ }^{7}$ show that vortex structures similar to the LEV and TEV form. It is therefore postulated that the circulatory and non-circulatory terms of the low order model should still apply at an angle of incidence of $90^{\circ}$, yet the Wagner function should not describe vortex growth. This article presents flow visualisation, PIV and force measurements to provide evidence of contributing factors for the growth of the LEV and TEV, as well as challenge the formulation of the low order model.

\section{A. Low Order Model at $90^{\circ}$}

This section describes modifications made to the low order model relevant for a surging wing at $90^{\circ}$ incidence. For a surging wing the low order model described by Babinsky et al. ${ }^{3}$ treats the flow as two dimensional, and deconstructs the lift force into an added mass component, and a circulatory component accounting for growth and advection of the leading and trailing edge vortices. At $90^{\circ}$ incidence the flow is initially symmetric about the mid plane of the wing, thus the net force on the wing will act in the direction of drag (X direction in figure 1).



Figure 1: Schematic of a surging wing

Vorticity shed at the wing edges is modelled as an inviscid vortex pair, as shown in figure 2a. Meanwhile the added mass component is calculated from the inviscid flow about a flat plate, as shown in figure $2 \mathrm{~b}$. The added mass force $\left(F_{n o n-c i r c}^{\prime}\right)$ per unit span, in the direction normal to the chord of the aerofoil is given by equation (1). The mass term in equation (1) is equivalent to the mass of fluid contained in a circular cylinder with a diameter equal to 1 chord $(c)$.

$$
F_{n o n-c i r c}^{\prime}=\overbrace{\frac{\rho \pi c^{2}}{4}}^{\text {mass }} \overbrace{\dot{U}^{\text {accel. }}}^{\text {and }}
$$

When non-dimensionalised with respect to the steady state free stream $\left(U_{\infty}\right)$, the added mass force coefficient is:

$$
C_{n o n-c i r c}=\frac{\pi c}{2 U_{\infty}^{2}} \dot{U}
$$




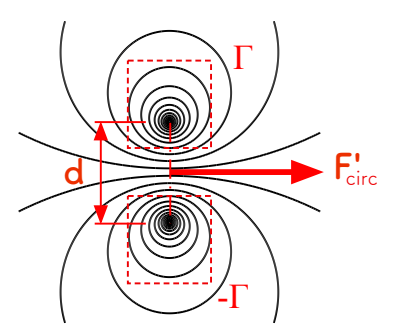

(a) Circulatory force (production and advection of vortex pair)

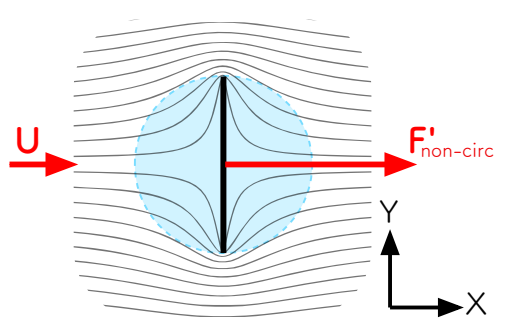

(b) Non-circulatory force (virtual mass)

Figure 2: Low order model force components.

The circulatory force is taken from Lamb, ${ }^{8}$ who expressed the net momentum $(\mathrm{J})$ of a flow for a vortex pair (equation 3).

$$
J=\rho \Gamma d
$$

The time derivative gives the circulatory force $\left(F_{\text {circ }}^{\prime}\right)$ in the drag direction:

$$
F_{\text {circ }}^{\prime}=\rho(\dot{\Gamma} d+\Gamma \dot{d})
$$

Which in non-dimensional form becomes:

$$
C_{c i r c}=\frac{2}{U_{\infty}^{2} c}(\dot{\Gamma} d+\Gamma \dot{d})
$$

Equation (4) shows that the circulatory force is dependent on the centre distance $(d)$ between the vortex pair, the rate of growth of circulation $(\dot{\Gamma})$, circulation strength $(\Gamma)$, and the relative rate of advection $(\dot{d})$. The added mass force is dependant only on acceleration rate $(\dot{U})$. The methodology described in the subsequent section is designed facilitate accurate measurement of the aforementioned variables, thus allowing deconstruction of the total drag force coefficient (equation 6) into circulatory and added mass components.

$$
C_{D}=C_{n o n-c i r c}+C_{c i r c}=\frac{\pi c}{2 U_{\infty}^{2}} \dot{U}+\frac{2}{U_{\infty}^{2} c}(\dot{\Gamma} d+\Gamma \dot{d})
$$

\section{Experimental Methodology}

This investigation utilised the Cambridge University Engineering Department (CUED) towing tank facility. The surge experiments were performed in the water tank to take advantage of Reynolds number scaling effects, minimise free-stream turbulence, facilitate flow visualisation, and suspend seeding particles for PIV. Firstly, flow visualisation was performed to qualitatively identify vortex structures. Force measurements were later taken to quantify the drag response of the wing. Finally, PIV measurements were taken to quantify the circulation and positions of detached vortices.

\section{A. Facility}

A schematic of the CUED towing tank is shown in figure 3. The tank is $7 \mathrm{~m}$ in length, has a width of 1 $\mathrm{m}$, and a maximum depth of $0.99 \mathrm{~m}$. For the experiments in this study the water level is $0.80 \mathrm{~m}$. A carbon fibre wing is used with length $0.48 \mathrm{~m}$, chord $0.12 \mathrm{~m}, 2 \%$ thickness. The wing was mounted to a servo driven carriage that traverses longitudinally down the tank. A skim plate was fitted between the wing and free surface of the water with a gap of $3 \mathrm{~mm}$ to the upper wing tip. Due to symmetry, the skim plate gives the wing an approximate aspect ratio of 8 . A clear perspex section in the centre of the tank allows cameras and a laser sheet to be positioned on both the sides, and underneath the test section for PIV and flow visualisation. 


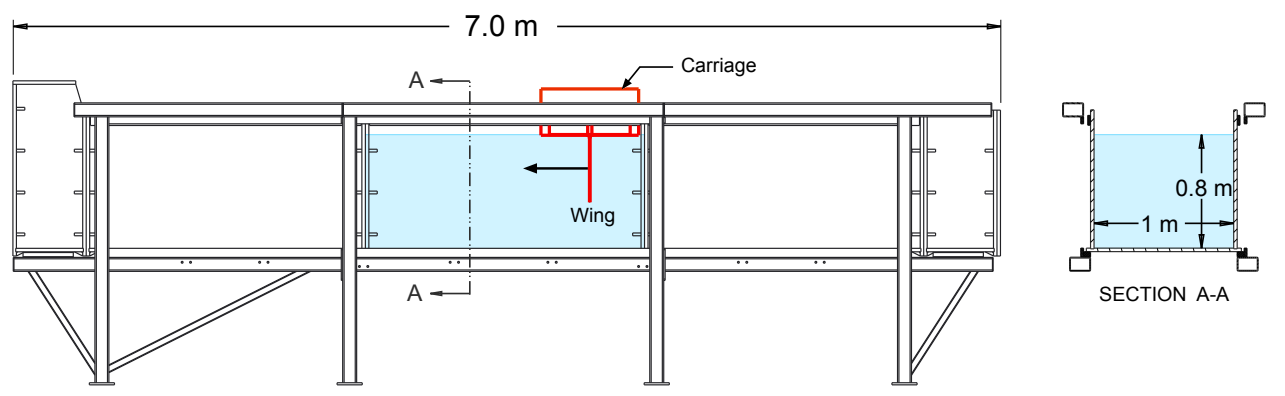

Figure 3: CUED towing tank and wing rig.

\section{B. Sensors and Data Acquisition}

Forces are acquired using a Flow Dynamics two component load cell. The load cell has a maximum rated capacity of $\pm 50 \mathrm{~N}$, and resolution of $0.01 \mathrm{~N}$. Calibration is performed by incrementally loading and unloading the load cell with known masses. Carriage position is measured using an electro-optical sensor mounted to the carriage, and an interrupt strip with a resolution of $4 \mathrm{~mm}$ (alternating black and white striped tape) running longitudinally down the length of the tank. The velocity and acceleration of the carriage was calculated by numerically differentiating the carriage position measurements. Both force and position data was recorded using LabView and a National Instruments 14 bit data acquisition card at a frequency of $1 \mathrm{kHz}$.

To attenuate resonant vibrations in the test rig, force measurements are filtered using a zero phase shift moving average with a 100 sample window size. The window size corresponds approximately to 1 full period for the first mode vibration of the test rig. Each end region of the moving average is treated by incrementally decreasing window size. Force measurements are then averaged over 10 test runs. An example of the force signal for a series of 10 test runs, and the filtered average is shown in figure 4 . The sample has a standard error of approximately $1 \%$ relative to the peak mean force, indicating excellent repeatability between test runs. The filtered average removes the resonant test rig vibration, and mostly retains peaks of interest in the transient force history. All force measurements have been compensated for the inertial force due to the self mass of the wing. This was measured using empty tank runs with kinematics identical to the fast and slow surge test cases. The kinematics of each of the test cases is discussed next.

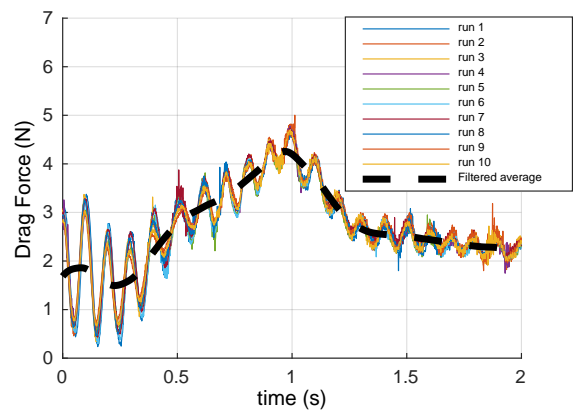

Figure 4: Typical drag force history and filtered sample average: $\operatorname{Re}=20,000$.

\section{Kinematics}

To distinguish inertial effects both a 'fast' case (acceleration over $1 \mathrm{c}$ ) and 'slow' surge case (acceleration over $6 \mathrm{c}$ ) were tested. Each profile consists of uniform acceleration of the wing, from rest, to a final free stream velocity $\left(U_{\infty}\right)$ through initially quiescent fluid. Thereafter the wing translates at steady speed. Ideal and measured kinematic profiles are shown in figure 5. It is important to note that constant acceleration 
with time gives a velocity profile proportional to the square root of position (s/c). Noise in the measured acceleration profiles is caused by the numerical differentiation of position sensor data.
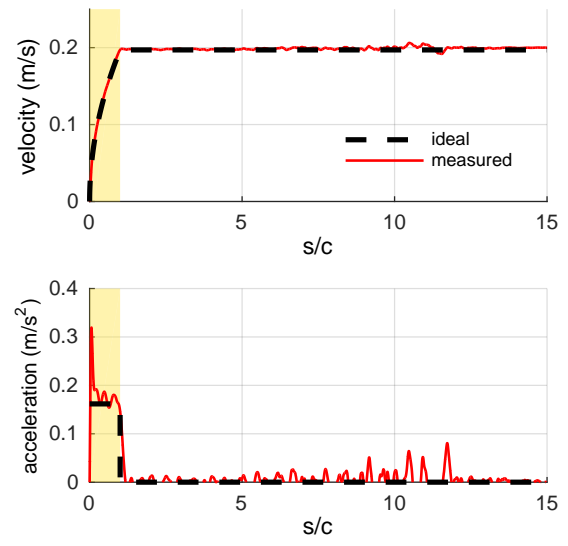

(a) acceleration over 1 chord


(b) acceleration over 6 chords

Figure 5: Ideal and measured velocity and acceleration profiles: Re $=20,000$. Velocity and acceleration profiles averaged over 10 runs. Acceleration region highlighted in yellow.

Force measurements were taken at a Reynolds number of 20,000, while dye flow visualisation and PIV measurements were taken using a Reynolds number of 10,000. The force measurements were taken at the higher Reynolds number to reduce the effect of measurement error due to rig vibration, while the lower Reynolds number for PIV and flow visualisation reduces difficulties with image acquisition. Prior studies have shown that the force coefficient has little dependance on Reynolds number within this range. ${ }^{4,9,10}$

\section{Dye Flow Visualisation}

To visualise flow topology, a milk and water dye mixture was injected into the flow through two tubes on the downstream face of the wing. Milk is used as it is approximately neutrally buoyant, retards diffusion of streak lines, and has good reflective properties. ${ }^{11}$ To minimally disrupt the flow, the dye lines were placed at the mid chord to correspond with the expected stagnation point on the wing. The setup is shown in figure 6a. Each injection point was located mid span of the wing, and approximately $4 \mathrm{~mm}$ from the wing edge. A syringe pump injected dye at a constant flow rate, such that dye seeped from each tube and did not overly disturb the flow. Figure $6 \mathrm{~b}$ shows the camera and lighting setup. The dye injected into the flow was illuminated by a light sheet of approximately $5 \mathrm{~mm}$ thickness cast horizontally across the test section. A Nikon DSLR camera photographed the flow from the underside of the test section.

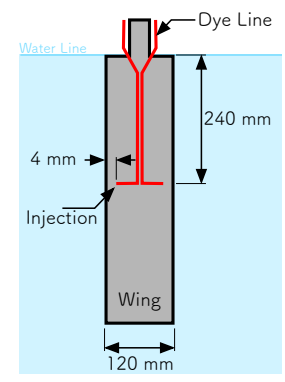

(a) Dye injection points

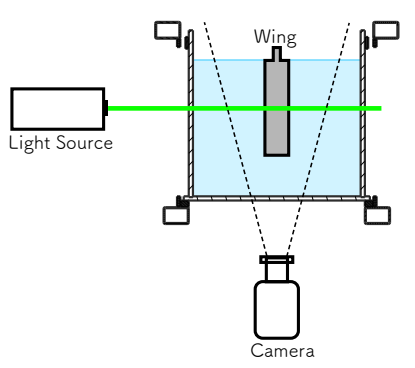

(b) Flow visualisation and PIV setup

Figure 6: Schematic of flow visualisation and PIV setup. 


\section{E. Particle Image Velocimetry}

PIV measurements were taken using a commercial LaVision Flowmaster 2D PIV system. Titanium Dioxide $\left(\mathrm{TiO}_{2}\right)$ particles were illuminated using a Nd:YLF $527 \mathrm{~nm}$ wavelength laser. Images were captured using a single high speed camera at a resolution of $1280 \times 800$ pixels. Due to a minimum pulse rate for the laser, the images were captured at $320 \mathrm{~Hz}$ and later down sampled. The laser and camera were set up in the tank in a similar manner as for flow visualisation, as shown in figure $6 \mathrm{~b}$. To minimise shadow regions caused by the wing, the laser was aligned edge on to the wing when at $\mathrm{s} / \mathrm{c}=0$. The camera was positioned directly underneath the wing at this position to minimise the lower section of wing obstructing the camera view of the illuminated particle plane. This allows the full field of view to be resolved at $\mathrm{s} / \mathrm{c}=0$. PIV measurements were averaged over 5 runs, and processed with $32 \times 32$ pixel interrogation windows and a $50 \%$ overlap. According to Raffel et al., ${ }^{12}$ random error in PIV measurement can be caused by variation in seeding particle diameter, mean particle shift, and seeding density. For the current experiment these have been estimated as 0.03 , 0.01 and 0.025 pixels respectively. The total random error is therefore estimated to be 0.065 pixels, which corresponds to $3 \%$ of the free stream velocity.

\section{Vortex Detection}

Vortices were detected using the non-local $\gamma_{2}$ technique described by Graftieaux et al. ${ }^{13}$ and Morgan et al. ${ }^{14}$ The $\gamma_{2}$ method, given by equation (7), and illustrated in figure 7 , quantifies the swirl coherence of velocity vectors about the centre of a moving interrogation window (of area $s$, containing $N$ points). As only the direction of velocity vectors is considered, the method can be used to determine the location of vortices, but not their strength. From figure 7 it can be seen that at the centre of an isolated vortex the angle $(\theta)$ between the velocity and position vector relative to the centre of the interrogation window is $90^{\circ}$, thus $\gamma_{2} \approx 1$. In this investigation the vortex centre was assumed to be the centroid of the $\gamma_{2}=2 / \pi$ contour. This is approximately the vortex core.

$$
\gamma_{2}=\frac{1}{N} \sum_{S} \sin \theta
$$

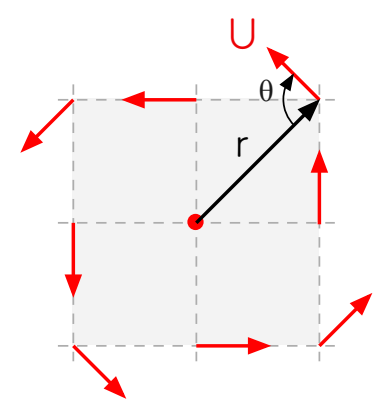

Figure 7: $\gamma_{2}$ schematic

\section{Circulation Measurement}

Circulation, $\Gamma(r)$, was calculated from the detected vortex centres using concentric circular lines of integration up to 2.5 core radii. A linear interpolation was used both between each measurement velocity vector, and in correction of incomplete circular integral lines due to obstructed camera field of view. The $\Gamma(r)$ measurements were fit to an ideal Lamb-Oseen vortex, as described by equation (8), to estimate the total circulation $\Gamma_{0}$ and core radius $r_{c}$. The method was developed by Stevens, ${ }^{15}$ who showed using an isolated synthetically generated vortex that gaussian noise with a standard deviation of $20 \%$ has negligible influence on the measured vortex circulation and core radii. The accuracy of the method is discussed next.

$$
\Gamma(r, t)=\Gamma_{0}\left(1-e^{\left(-r^{2} / r_{c}^{2}\right)}\right)
$$




\section{Vortex Proximity Error Correction}

Measurement of the circulation of a Lamb-Oseen vortex in isolation can differ to the identical measurement when taken in close proximity to one or more other vortices. Since circulation is equivalent to the flux of vorticity $\left(\Gamma=\int_{A} \omega \cdot d A\right)$, measured circulation varies because additional vorticity is superimposed onto the area of integration. The measured centre of each vortex using the $\gamma_{2}$ method is additionally shifted due to a non-linear velocity gradient induced across each $\gamma_{2}$ interrogation window. To demonstrate this effect, an artificially generated pair of Lamb-Oseen vortices separated by 2.5 core radii $\left(d / r_{c}=2.5\right)$ is shown in figure 8. The centre separation distance of the two vortices, as calculated from the centroid of the $\left|\gamma_{2}\right|=2 / \pi$ contours (shown), is notably greater than the true centre distance. The measured circulation shown in figure $8 \mathrm{~b}$ significantly deviates from the true profile (for an isolated vortex) for $r / r_{c} \geq 1$. This is due to increasing inclusion of vorticity of the adjacent vortex as the surface of integration grows in diameter, and results in an approximate $30 \%$ underestimation of $\Gamma_{0}$ (refer to figure 10).

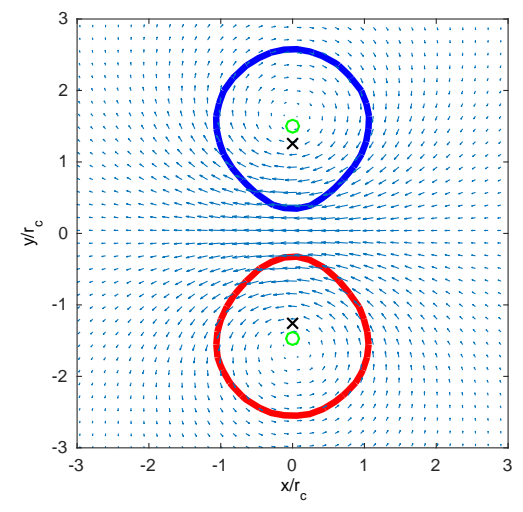

(a) Velocity with $\gamma_{2}$ contours. Black cross $=$ true centre. Green circle $=$ measured centre

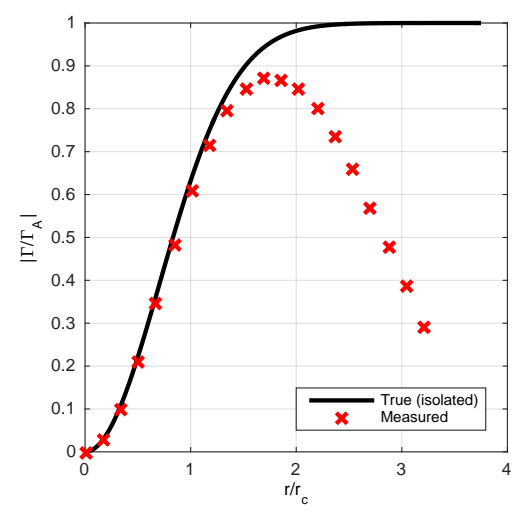

(b) Measured and true circulation with non-dimensional radius

Figure 8: Uncorrected measurement of an artificially generated Lamb-Oseen vortex pair.

To alleviate the influence of adjacent vortices, an iterative velocity field correction similar to that described by Morgan et al. ${ }^{14}$ was implemented. First the approximate centre and circulation of each vortex was calculated using the method described in sections 1 and 2 . The velocity fields for ideal Lamb-Oseen vortices was subtracted from the measured velocity field the for all vortices except one. The centre and circulation of the now isolated vortex was recalculated. Finally the procedure was repeated for all vortices in the flow field, and multiple iterations performed to refine accuracy. Figure 9 shows the measured vortex centres and circulation after 3 correction iterations for the artificially generated Lamb-Oseen vortex pair. The measured vortex centres almost exactly match the true centres, while the circulation distribution fits within $3 \%$ of the true profile.

The errors incurred if the velocity field from neighbouring vortices is uncorrected for, and corrected using 3 iterations is shown in figure 10. For 3 velocity field corrections there is negligible error between the measured and true centre distance for vortices separated by greater than 3 core radii (figure 10a). The uncorrected measurements, however, show significant error. Circulation with relative vortex separation is shown in figure 10b. Similarly, with 3 velocity field corrections there is negligible error in measured circulation for separation distances greater than 3 core radii. All PIV measurements have been processed with 3 velocity field correction iterations. 


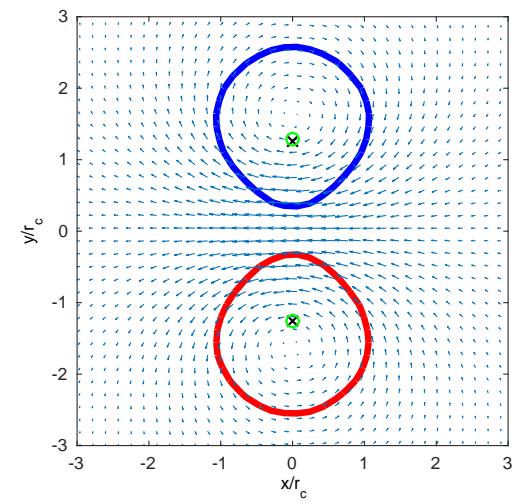

(a) Velocity with $\gamma_{2}$ contours. Black cross $=$ true centres. Green circle $=$ measured centres



(b) Circulation with radius from centre

Figure 9: Corrected measurement of an artificially generated Lamb-Oseen vortex pair



(a) Measured vortex centre separation

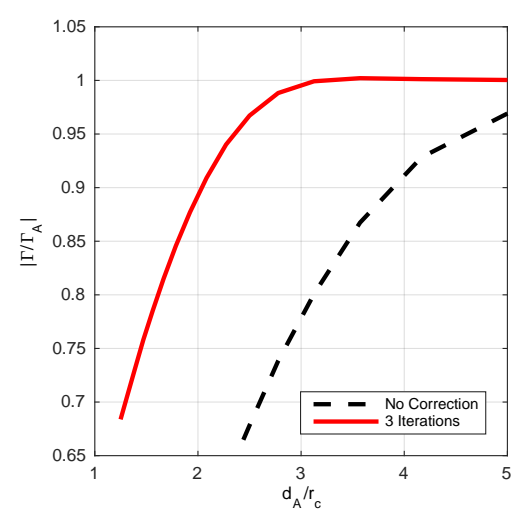

(b) Measured Circulation

Figure 10: Measurement error with relative vortex separation distance. Vortex centre distance and circulation are normalised with respect to the true artificially generated values.

\section{Dye Flow Visualisation}

To qualitatively evaluate differences in flow topology, dye flow visualisation of the fast and slow surge cases is first compared. Figure 11 shows the dye flow visualisation at positions of $\mathrm{s} / \mathrm{c}=0.50,1$, and 1.25 respectively. In the 'fast' case, these positions capture the acceleration phase, end of the acceleration phase, and steady state. For the slow surge all 3 images occur during the acceleration phase. For both the fast and slow surge cases a counter-rotating pair of vortices is visible, similar to that assumed by the circulatory component of the low order model. The distinct pattern of a Kelvin-Helmholtz flow instability leading from the wing edges to each vortex is also visible, indicating the presence of an unstable shear layer. The length between each roller (wavelength) is noticeably longer for the slow surge case, which is potentially attributed to a lower shear rate. It is unlikely that the rollers are caused by vibration of the plate. Vibration at the resonant frequency would cause a shorter wavelength for the slow surge case, and longer in the fast case. This is opposite to that observed in the flow visualisation.

On average, the flow topology is remarkably similar between the fast and slow surge cases despite significant differences in velocity (refer to figure 5). The distance between the vortex centres is approximately equal to one chord for both cases. This implies that the rate of advection $\dot{d}$ is low, and therefore the force component due to vortex advection may be negligible (refer to equation 5 ). For each s/c position the vortices 
appear to have moved the same distance downstream relative to the wing. Any differences in force at any given position between the fast and slow cases are therefore unlikely to be caused by a significant difference in flow topology.

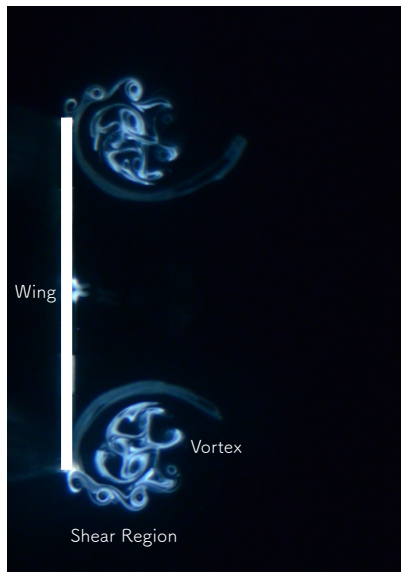

(a) Fast surge: $\mathrm{s} / \mathrm{c}=0.50$

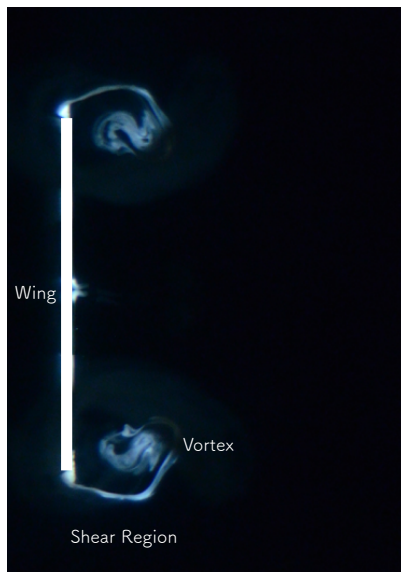

(d) Slow surge: $\mathrm{s} / \mathrm{c}=0.50$



(b) Fast surge: $\mathrm{s} / \mathrm{c}=1.0$

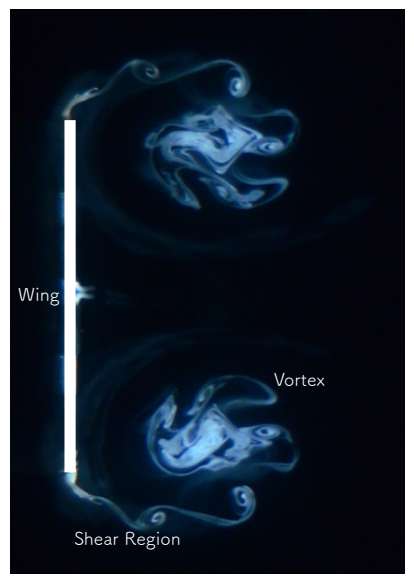

(e) Slow surge: $\mathrm{s} / \mathrm{c}=1.0$

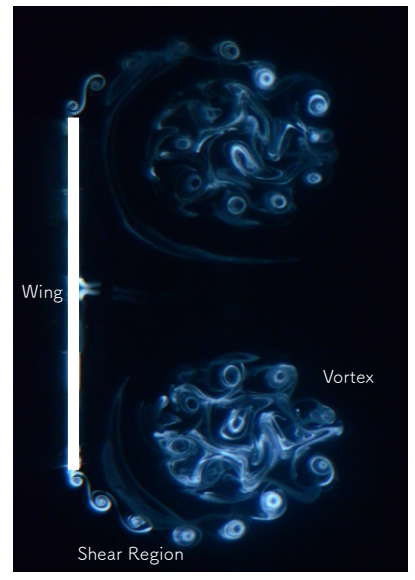

(c) Fast surge: $\mathrm{s} / \mathrm{c}=1.25$

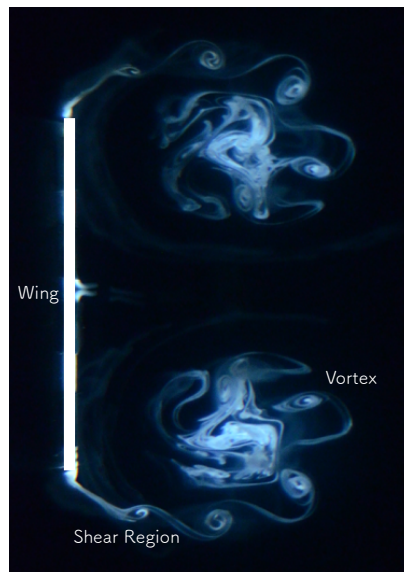

(f) Slow surge: $\mathrm{s} / \mathrm{c}=1.25$

Figure 11: Flow visualisation showing the formation of two counter-rotating vortices for the fast and slow surge cases at $\mathrm{s} / \mathrm{c}=0.50,1.0$, and 1.25 . The top row shows the fast surge case, while the bottom row shows the slow surge case. Flow direction: left to right.

\section{Particle Image Velocimetry}

The observed similarity in flow topology is confirmed by PIV measurement. The flow fields for $\mathrm{s} / \mathrm{c}=0.25$, 1 and 2 are shown in figure 12 for the fast and slow cases. Here the $\gamma_{2}=2 / \pi$ contours, and vortex centres (green circles) are superimposed onto the velocity vectors. The measurements are displayed in the frame of reference of an observer fixed relative to the fluid, i.e. the wing translates from $\mathrm{s} / \mathrm{c}=0$. As before, striking similarity between the fast and slow surge cases can be observed. It is important, however, to note that the time scales differ between fast and slow cases. Vortex strength, or rate of growth between the fast and slow cases, is likely not equivalent (the magnitude of the velocity vectors in figure 12 are scaled arbitrarily). From figure 12 the following is visible:

- A clockwise rotating vortex grows from the upper wing edge, and a complementary anti-clockwise vortex forms at the lower edge. Visually the core contours are also approximately equivalent.

- The vortex centres are separated by approximately one chord for all s/c positions. 
- The vortex centres advect downstream relative to the wing. Between the fast and slow surge cases the rate of advection is such that the vortices are approximately equivalently located for any s/c position. This implies that vortex position is directly related to wing position.

- The radii of each $\gamma_{2}=2 / \pi$ contour appears marginally larger for the fast surge case compared to the slow case. For $s / c=2$ the $\gamma_{2}$ contour of the slow surge case is quite distorted by the shear layer feeding each vortex.

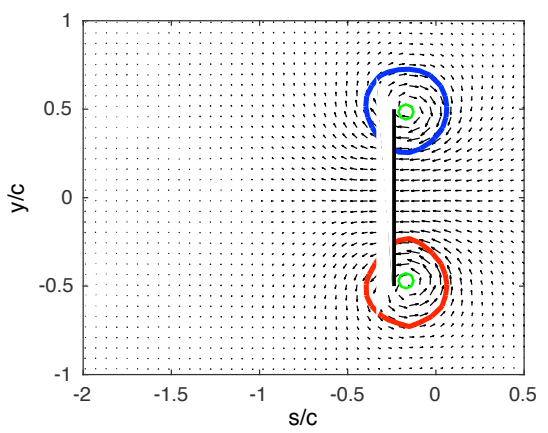

(a) Fast surge: $\mathrm{s} / \mathrm{c}=0.25$

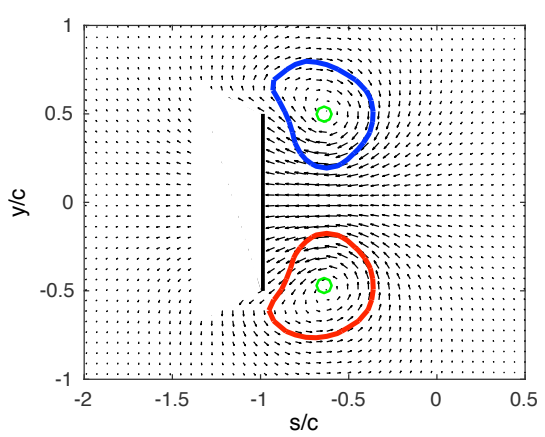

(b) Fast surge: $\mathrm{s} / \mathrm{c}=1$

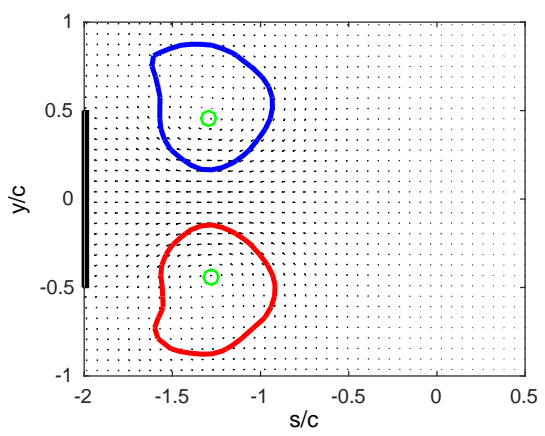

(c) Fast surge: $\mathrm{s} / \mathrm{c}=2$

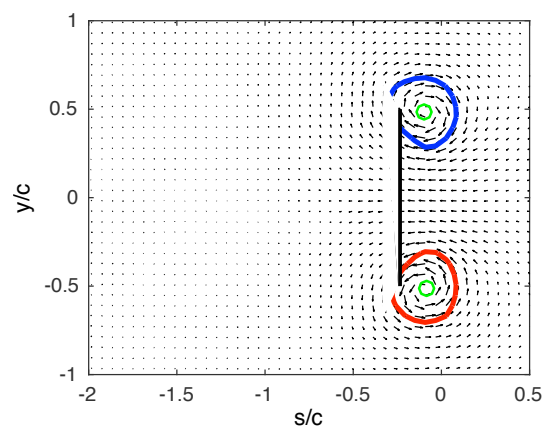

(d) Slow surge: $\mathrm{s} / \mathrm{c}=0.25$

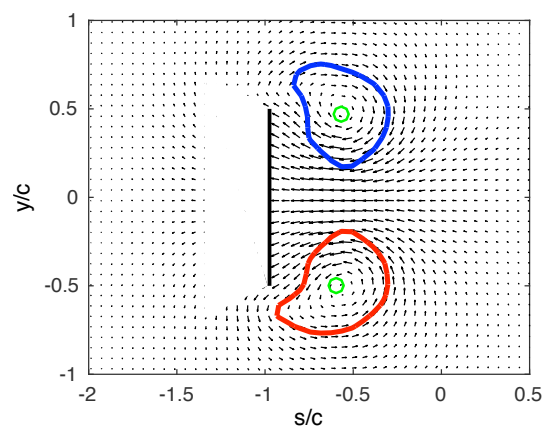

(e) Slow surge: $\mathrm{s} / \mathrm{c}=1$



(f) Slow surge: $s / c=2$

Figure 12: PIV measurements and $\gamma_{2}=2 / \pi$ contours for the fast and slow surge cases. 


\section{Vortex Tracking}

From the PIV measurements, the positions of the vortices is now sequentially tracked. Figure 13a shows the streamwise distance between the wing and vortex centres. The vortices appear to advect approximately linearly from the wing, at a rate of $\Delta x / s=0.37$. This is consistent with observation from the flow visualisation in section III, that vortex position is directly dependant on wing position (there is little difference between the fast and slow surge cases). Figure 13b shows the vortex centre separation distance $(d)$. The vortex centres remain separated by approximately 0.90 chord lengths for the measured s/c range. As explained in section 3, the detected location of the vortex centres is influenced by the flow field induced by adjacent vortices. On figure 13b, the uncorrected centre separation distance has been shown for comparison. The uncorrected separation distance shows the vortices gradually drift apart (due to increasing core radii), leading to an approximate error of $20 \%$ at s/c $=2.25$.

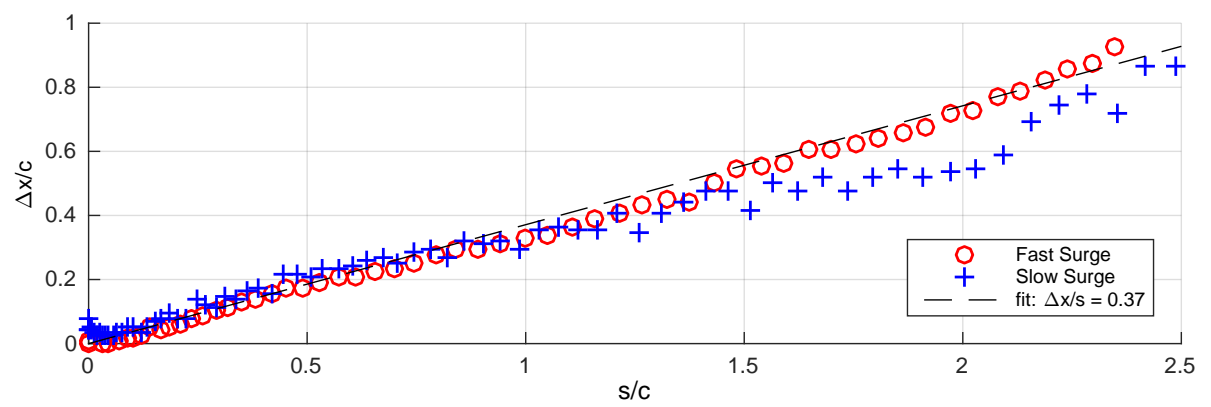

(a) $\mathrm{X}$ direction: wing to vortex centre distance $(\mathrm{s} / \mathrm{c}-\mathrm{x} / \mathrm{c})$

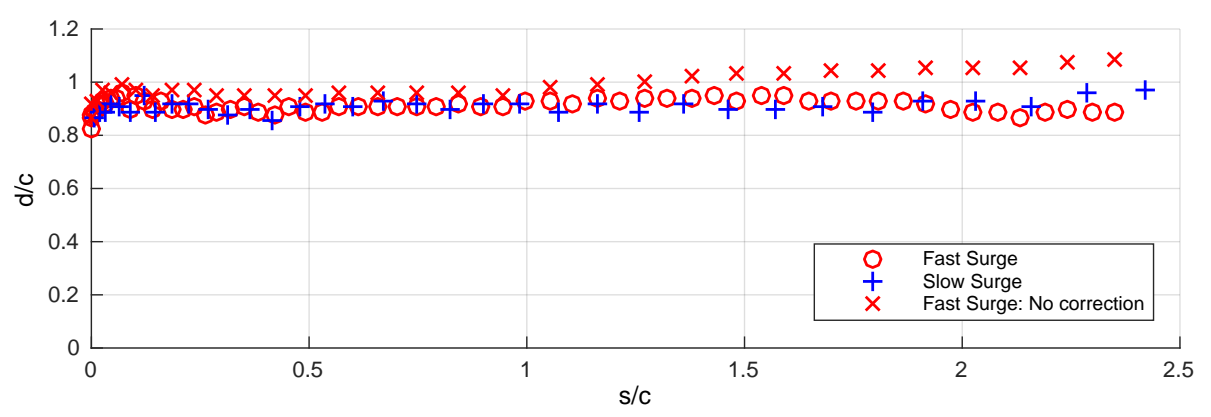

(b) Y direction: vortex centre separation

Figure 13: Vortex trajectories with wing position for the fast an slow surge cases.

These observations allow the circulatory term of the low order model $\left(C_{c i r c}=\frac{2}{U_{\infty}^{2} c}(\dot{\Gamma} d+\Gamma \dot{d})\right)$ to be simplified. Since the distance between the vortex centres is approximately constant, $\dot{d}$ is approximately equal to zero, and $d \approx c$. The circulatory force coefficient is therefore reduced to:

$$
C_{\text {circ }}=\frac{2}{U_{\infty}^{2}} \dot{\Gamma}
$$

This states that the circulatory force coefficient is proportional to the rate of change of circulation. If it is assumed that equation (9) remains valid throughout the motion of the wing (and thus well into steady state), one might conclude that the Wagner function can not correctly describe the vortex growth for large $\mathrm{s} / \mathrm{c}$ positions. Here Wagner's function asymptotes to $\dot{\Gamma}=0$, and since the added mass force vanishes, the total drag coefficient prediction of low order model (equation 6) is zero. As we expect there to be finite drag, components of the low order model, or the use of the Wagner function is unphysical. To investigate this problem, measurements of vortex circulation are next considered. 


\section{Circulation}

Circulation for the fast and slow surge cases is shown in figure 14. The Wagner function, and the modified Wagner function have been calculated assuming that $\Gamma$ follows the Wagner prediction for bound circulation. The asymptotic value is given by $\Gamma=c U_{\infty} \pi \alpha$, which is derived from linearised thin airfoil theory $\left(C_{l}=2 \pi \alpha\right)$. As mentioned previously, the modified Wagner function compensates for a non-impulsive start by scaling the Wagner function by the ratio of the instantaneous to steady state velocity.

From figure 14 the following is observed:

- During the acceleration regime of the fast surge case, the circulation approximately increases linearly with $\mathrm{s} / \mathrm{c}$ at a rate $d \Gamma / d s=0.25$ (there is subtle curvature). During steady translation the growth rate does not asymptote towards zero (as predicted by the Wagner function), but rather continues to increase linearly at a reduced rate of $d \Gamma / d s=0.12$.

- During the acceleration regime of the slow surge case, the circulation also approximately increases linearly with $\mathrm{s} / \mathrm{c}$, however, at a comparatively lower rate of $d \Gamma / d s=0.10$. This is similar to that of the fast surge case when in steady translation.

- Somewhat surprisingly, the circulation closely fits the modified Wagner function for both the fast and slow surge cases during acceleration.



(a) Fast surge

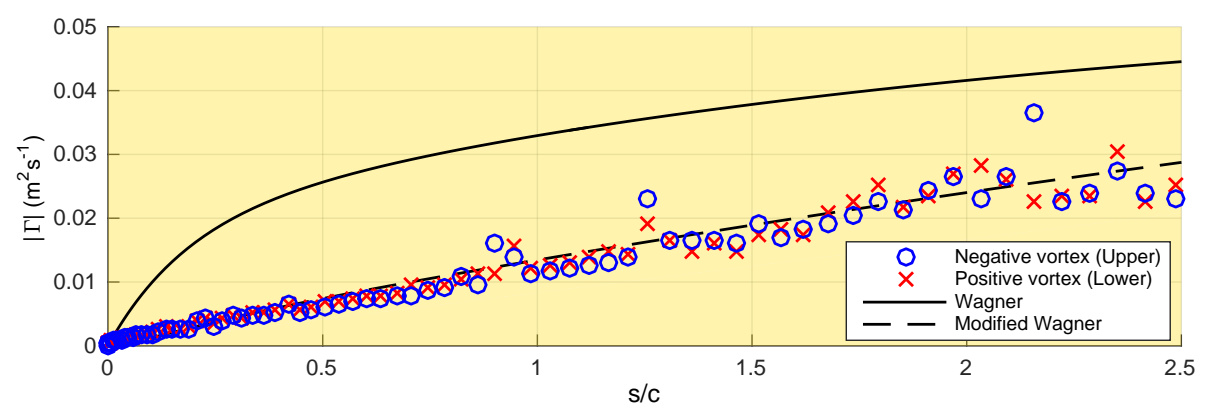

(b) Slow surge

Figure 14: Circulation with s/c for the fast and slow surge cases. Wagner's function, and the modified Wagner function is included for reference.

Firstly, the reader is reminded that a constant $\dot{\Gamma}$ term after acceleration is consistent with there being constant drag. In figure 14a we observed a linear gradient for the fast case for $s / c>1$, which would result in a constant circulatory force (as the wing is travelling at constant velocity, $\dot{\Gamma}=\frac{d \Gamma}{d s} \cdot \frac{d s}{d t}$ ). Surprisingly, the change in gradient at $s / c=1$ (fast case) indicates that the circulatory force has reduced in magnitude at the cease of acceleration. The circulatory force thus appears dependant on acceleration rate. This contradicts the low order model which includes a separate acceleration dependant force component, added mass. The link between added mass, generation of vorticity, and thus the measured circulation is discussed next. 


\section{Vorticity Production and Added Mass}

The change in growth rate of circulation can be explained by comparison of potential flow and PIV measurement immediately after the start of motion, as shown in figure 15 for the fast case. Here the measured flow field looks similar to potential flow. At $s / c=0.01$ the force response of the fast surge case, and thus the change in impulse of the flow field, is dominated by the effect of virtual mass. This force is also present in an accelerating potential flow, where the wing can be represented by a vortex sheet. This sheet varies both spatially and with time $\gamma(y, t)$, and is distributed in a self similar manner $k(y)$, such that there is zero flow through the sheet. The magnitude of the sheet is proportional to the incident velocity $U(t)$. We can therefore write:

$$
\gamma(y, t)=k(y) U(t)
$$

Physically, the strength of the vortex sheet represents vorticity contained in the shear layer forming along the upstream and downstream plate surfaces in a viscous flow. From equation (10), acceleration of the plate changes the vortex sheet strength, and thus in a viscous flow generates vorticity in the shear layer. From figure $15 \mathrm{~b}$ we see that the vorticity is concentrated at the upper and lower plate edges. If we subtract the free stream velocity so we are in the accelerating plate frame of reference (figure 15d), the resulting streamline pattern closely resembles a potential point vortex pair (figure 15c). It is therefore reasonable to assume that the procedures used to determine the strength of circulation of the vortices forming at the wing edges would additionally include the impulse due to added mass.



(a) Potential flow: Flat plate



(c) Potential flow: Vortex pair

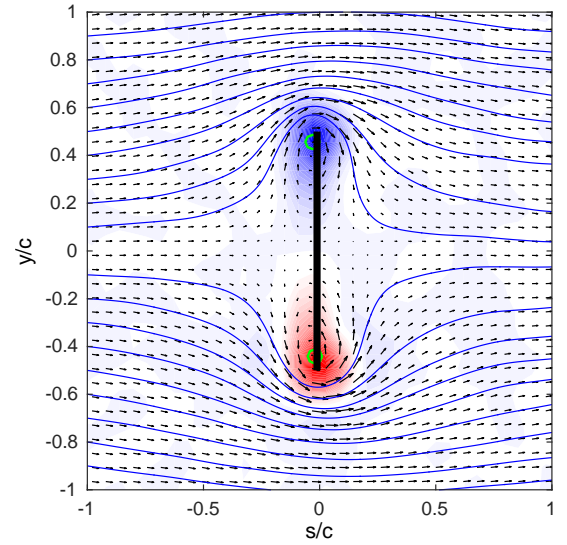

(b) PIV: Fixed plate reference



(d) PIV: Accelerating plate reference

Figure 15: Comparison of streamlines between potential flow and PIV measurement of the fast surge case at $\mathrm{s} / \mathrm{c}=0.01$. Coloured regions show normalised vorticity. 
An exact solution for the circulation due to added mass $\left(\Gamma_{m}\right)$, can be found from potential theory by taking the line integral of the vortex sheet across one half of the plate. Alternatively this can be approximated by equating the circulatory and non-circulatory drag coefficient components of the low order model (equations 2 and 9 respectively). While not derived here, the exact solution is:

$$
\Gamma_{m}=c U(t)
$$

The rate of change of circulation due to added mass is therefore:

$$
\dot{\Gamma}_{m}=c \dot{U}(t)
$$

The calculated circulation due to added mass for each test case is shown in figure 16. During the acceleration region of the fast case, the circulation is proportional to $\sqrt{s / c}$, and is constant during the steady state region. A discontinuous change in gradient at $s / c=1$, similar to that described in section 2 is seen. As the wing is undergoing constant acceleration for the slow surge case, we see no discontinuity in gradient.

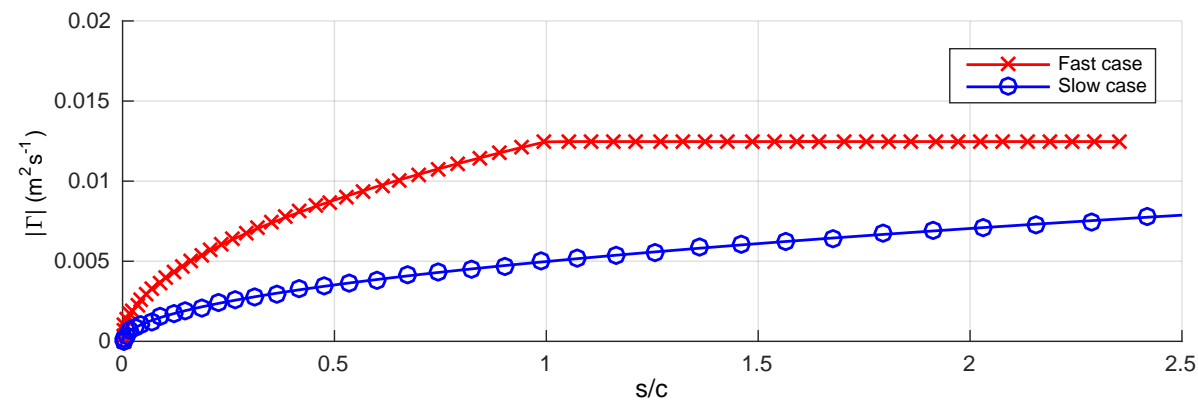

Figure 16: Added mass circulation with position.

If the circulation due to added mass is subtracted from that calculated using PIV measurements of the flow field, then the remainder is likely caused by other vorticity production mechanisms such as viscous shearing. The net circulation is shown in figure 17. For the measured range, the remaining circulation for the fast and slow surge cases appears to increase in proportion to $s / c$, at rates of 0.13 and $0.065 \mathrm{~m} / \mathrm{s}$ respectively. Notably, there is no apparent change in gradient at the end of the acceleration region of the fast surge case. This indicates that the vorticity generated due to added mass is accounted for.

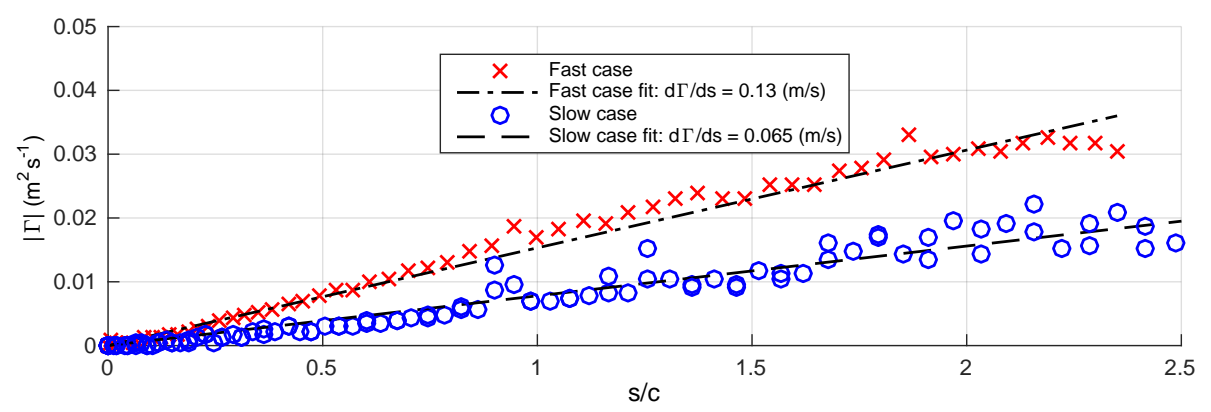

Figure 17: Circulation less the added mass component.

Since the gradient $(d \Gamma / d s)$ differs for the fast and slow surge cases, there is likely to be an additional dependance between circulation and acceleration rate. The exact relation of this dependance is unknown at present. If we consider the steady state (in absence of vortex shedding which is outside the scope of this discussion), circulation of the vortices could grow to such strength such that the self induced velocity of the vortex pair equals the free stream velocity. Here the vortices would cease to advect relative to the wing. To maintain steady state (and constant drag force) the vorticity shed at the plate tips must either dissipate, or cancel due to merging of the vortex pair. The measured drag force, and that calculated with the low order model are considered next. 


\section{Force Measurement}

Having removed the influence of added mass on circulation determined from PIV measurement, the force response of the wing can now be predicted using the low order model. The net circulatory force has been calculated using the linear fits given in figure 17, and the reduced circulatory component of the low order model (equation 9). Added mass was calculated with equation (2). This has been compared to direct force measurement for the fast and slow surge cases in figures 18 and 19 respectively.

Fast surge case: At $\mathrm{s} / \mathrm{c}=0$, force measurements show an initial drag coefficient of approximately 1 . During the acceleration region the drag coefficient increases to $C_{D}=3.5$, and drops sharply by $\Delta C_{D} \approx 1.5$ at the end of the acceleration region $(\mathrm{s} / \mathrm{c}=1)$. During steady translation, the drag force coefficient settles to approximately $C_{D}=1.8$, until s/c $\approx 7$, where it drops to $C_{D}=1.6$. Force coefficient inferred from the low order model reasonably closely resembles direct force measurement. The drop in drag coefficient at the end of the acceleration region is, however, underestimated, and the steady state drag coefficient is overestimated. Given the noise in circulation calculated from PIV measurements, uncertainty in carriage kinematics, and approximations made by the low order model, this error is not unreasonable.



Figure 18: Drag coefficient with position for the fast surge.

Slow surge case: At $\mathrm{s} / \mathrm{c}=0$, force measurements show a small initial offset in drag coefficient of $\Delta C_{D}=0.25$. During the acceleration region, the drag coefficient approximately increases linearly with position. At the end of the acceleration region $(\mathrm{s} / \mathrm{c}=6)$, the drag coefficient drops by $\Delta C_{D} \approx 0.25$, followed by a steady state drag coefficient of $C_{D} \approx 1.6$. Similar to the fast surge case, the force coefficient inferred from the low order model and PIV measurement reasonably closely resembles direct force measurement.

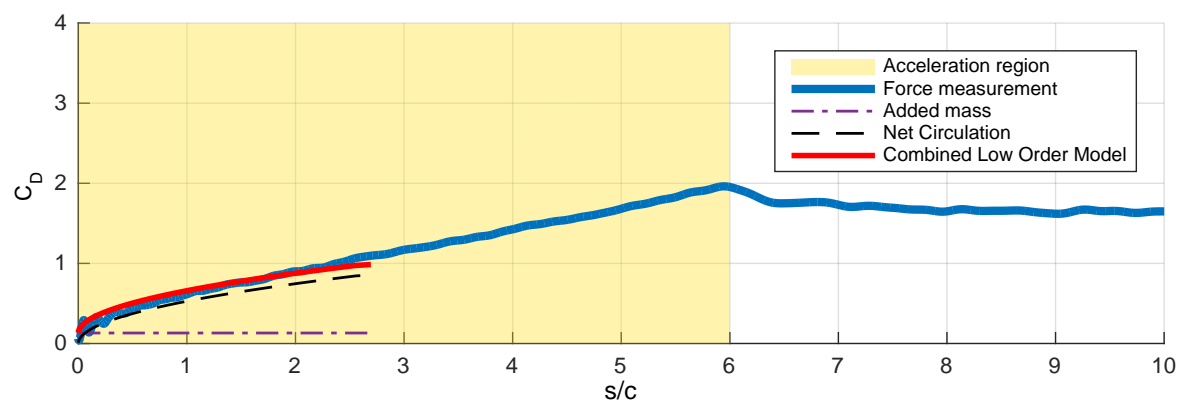

Figure 19: Drag coefficient with position for the slow surge.

Regardless of acceleration rate, the force coefficient predicted by the low order model closely matches direct force measurement. This suggests that the low order model, with the circulatory component adjusted for added mass, captures the force production mechanisms for a surging at $90^{\circ}$ incidence.

\section{Conclusions}

A simple surge experiment on a flat plate wing at $90^{\circ}$ incidence was performed to test a low order model developed by the NATO AVT-202 task group. The model deconstructs the force response of surging and 
pitching wings at high incidence into non-circulatory added mass components, and circulatory components that account for the dynamics of a leading and trailing edge vortex. In particular, the validity of a modified Wagner function for predicting LEV and TEV growth was reviewed.

The experimental investigation has shown:

- A vortex pair similar to a LEV and TEV form behind a surging wing at $90^{\circ}$ incidence. The physics captured by the low order model should therefore be applicable to this case.

- Measurements of the circulation of the vortex pair is remarkably similar to the modified Wagner function for $s / c \leq 2.5$, assuming steady state circulation is found from linearised thin airfoil theory $\left(C_{L}=2 \pi \alpha\right)$. It is unknown why this is the case.

- For a plate surging at $90^{\circ}$ incidence, the vortex centres are separated by a constant distance of approximately one chord length for $s / c \leq 2.5$. The circulatory force coefficient is therefore approximately proportional to the rate of change of circulation $(\dot{\Gamma})$.

- Contrary to the Wagner function, measurements and the low order model suggest that $\dot{\Gamma}$ does not diminish at steady state, thus giving non-zero drag.

- It is proposed that added mass is a mechanism that generates vorticity, and can be quantified from PIV measurements as a change in circulation.

- Reasonable agreement between the low order model with PIV measurement, and force measurement has been shown once the vorticity generated by added mass is correctly accounted for.

\section{Acknowledgments}

The author would like to acknowledge the Cambridge Commonwealth European and International Trust, Churchill College, and Schlumberger Limited for providing financial support.

\section{References}

${ }^{1}$ Davis, W. R. J., Kosicki, B. B., Boroson, D. M., and Kostishack, D. F., "Micro Air Vehicles for Optical Surveillance," the Lincoln Laboratory Journal, Vol. 9, No. 2, 1996, pp. 197-214.

${ }^{2}$ Ol, M. and Babinsky, H., "Extensions of Fundamental Flow Physics to Practical MAV Aerodynamics: TR-AVT-202," Tech. rep., North Atlantic Treaty Organization, 2016.

${ }^{3}$ Babinsky, H., Stevens, P. R. R. J., Jones, A. R., Bernal, L. P., and Ol, M. V., "Low Order Modelling of Lift Forces for Unsteady Pitching and Surging Wings." 54th AIAA Aerospace Sciences Meeting, jan 2016, pp. 1-12.

${ }^{4}$ Stevens, P. R. R. J., Babinsky, H., Manar, F., Mancini, P., Jones, A. R., Granlund, K. O., Ol, M. V., Nakata, T., Phillips, N., Bomphrey, R. J., and Gozukara, A. C., "Low Reynolds Number Acceleration of Flat Plate Wings at High Incidence," 54 th AIAA Aerospace Sciences Meeting, jan 2016, pp. 1-15.

${ }^{5}$ Pierce, D., "Photographic evidence of the formation and growth of vorticity behind plates accelerated from rest in still air," Journal of Fluid Mechanics, Vol. 11, No. 3, 1961, pp. 460-464.

${ }^{6}$ Pullin, D. I. and Perry, A. E., "Some flow visualization experiments on the starting vortex," Journal of Fluid Mechanics, Vol. 97, No. 2, 1980, pp. 239-255.

${ }^{7}$ Lian, Q.-X. and Huang, Z., "Starting flow and structures of the starting vortex behind bluff bodies with sharp edges," Experiments in Fluids, Vol. 8, 1989, pp. 95-103.

${ }^{8}$ Lamb, H., Hydrodynamics, Cambridge University press, 1895.

${ }^{9}$ Jones, A. R. and Babinsky, H., "Reynolds number effects on leading edge vortex development on a waving wing," Experiments in Fluids, Vol. 51, No. 1, 2011, pp. 197-210.

${ }^{10}$ Pitt Ford, C. W., Unsteady aerodynamic forces on accelerating wings at low Reynolds numbers., Ph.D. thesis, The University of Cambridge, 2013.

${ }^{11}$ Clayton, B. R. and Massey, B. S., "Flow visualization in water: a review of techniques," Journal of Scientific Instruments, Vol. 44, No. 1, 1967, pp. 2-11.

${ }^{12}$ Raffel, M., Willert, C. E., Wereley, S. T., and Kompenhans, J., Particle Image Velocimetry: A Practical Guide, 2nd Edition, Springer, 2007.

${ }^{13}$ Graftieaux, L., Michard, M., and Grosjean, N., "Combining PIV, POD and vortex identification algorithms for the study of unsteady turbulent swirling flows," Measurement Science and Technology, Vol. 12, No. 9, 2001, pp. 1422-1429.

${ }^{14}$ Morgan, C., Babinsky, H., and Harvey, J., "Vortex Detection Methods for Use with PIV and CFD Data," 47th AIAA Aerospace Sciences Meeting, American Institute of Aeronautics and Astronautics, jan 2009.

${ }^{15}$ Stevens, P. R. R. J., Unsteady Low Reynolds Number Aerodynamic Forces, Ph.D. thesis, University of Cambridge, 2013. 